\title{
Waveguide-Based Antenna Arrays for 5G Networks
}

\author{
Arismar Cerqueira Sodré Jr. $\mathbb{D}^{1},{ }^{1}$ Igor Feliciano da Costa $\left(\mathbb{D},{ }^{1}\right.$ Renan Alves dos Santos, ${ }^{2}$ \\ Hugo Rodrigues Dias Filgueiras $\mathbb{D}^{1},{ }^{1}$ and Danilo Henrique Spadoti $\mathbb{D}^{2}$ \\ ${ }^{1}$ Laboratory WOCA (Wireless and Optical Convergent Access), National Institute of Telecommunications (INATEL), \\ João de Camargo Avenue 510 P.O. Box 05, 37540-000 Santa Rita do Sapucaí, MG, Brazil \\ ${ }^{2}$ Federal University of Itajubá (UNIFEI), Benedito Pereira dos Santos Avenue 1303 P.O. Box 50, 37500-903 Itajubá, MG, Brazil
}

Correspondence should be addressed to Arismar Cerqueira Sodré Jr.; arismar@inatel.br and Igor Feliciano da Costa; igorfelicianocosta@gmail.com

Received 12 July 2017; Revised 10 November 2017; Accepted 23 November 2017; Published 4 February 2018

Academic Editor: Miguel Ferrando Bataller

Copyright (c) 2018 Arismar Cerqueira Sodré Jr. et al. This is an open access article distributed under the Creative Commons Attribution License, which permits unrestricted use, distribution, and reproduction in any medium, provided the original work is properly cited.

\begin{abstract}
This work reports the development of two high-performance waveguide-based antenna arrays for 5G cellular networks, operating in the underutilized millimetre wave (mm-wave) frequency spectrum. Two different scenarios of mm-wave communications are proposed for illustrating the applicability of the proposed arrays, which provide specific radiation patterns, namely, $12 \mathrm{dBi}$ gain omnidirectional coverage in the $28 \mathrm{GHz}$ band and dual-band sectorial coverage using the 28 and $38 \mathrm{GHz}$ bands with gain up to $15.6 \mathrm{dBi}$. Numerical and experimental results of the array reflection coefficient, radiation pattern, and gain have been shown in an excellent agreement.
\end{abstract}

\section{Introduction}

The fifth-generation (5G) cellular systems have been vastly investigated in the last years [1-5], as well as its applicability for the new internet services, such as high-definition video stream, bitpipe communications at Gbps [1], tactile internet, Internet of Things (IoT), rural access networks [4], and autonomous cars. New challenges, including spectrum, propagation channel, reliability, cost, and energy efficient aspects, become extremely important for the success of $5 \mathrm{G}$ networks. More specifically, it is envisioned throughputs of $10 \mathrm{Gbps}$ for enabling virtual reality and immersive experience, latency lower than $1 \mathrm{~ms}$ for making self-driving cars and intelligent traffic management become a reality in future cities and billions of connections due to the exponential growth of IoT.

The 5G networks are likely to operate in the centimetrewave $(3-30 \mathrm{GHz})$ and millimetre-wave $(30-300 \mathrm{GHz})$ frequency bands [2,3], in which there is a lot of unexploited spectrum worldwide. The E-band and $\mathrm{W}$-band have also been analyzed for fulfilling the tough requirements of this new generation [6]. However, those frequency ranges suffer from even higher free-space loss, increasing complexity, and cost to deploy access networks. There are plenty of studies underway in both industry and academia on characterization of frequencies below and above $30 \mathrm{GHz}$ for $5 \mathrm{G}$ applications. Rappaport et al. have recently claimed 28 and $38 \mathrm{GHz}$ frequency bands as promising solutions for $5 \mathrm{G}$ networks, since atmospheric absorption does not significantly contribute to additional path loss [1]. They draw this conclusion after conducting extensive propagation measurement campaigns at 28 and $38 \mathrm{GHz}$, with the purpose of obtaining insight on angle of arrival, angle of departure, RMS delay spread, path loss, building penetration, and reflection characteristics in $\mathrm{mm}$-wave cellular systems.

Particularly, in the context of antennas, $5 \mathrm{G}$ will demand a revolution, since they are going to be designed for short wavelengths (mm-waves), which easily fit in handsets, but are still long enough to bend around or penetrate obstacles, such as buildings and foliage. Additionally, mm-waves suffer from high attenuation due to rain. To overcome all these challenges, as well as free-space attenuation, different antenna types have been exploited for 5G networks. Most works published in literature on $5 \mathrm{G}$ antennas are concerning MIMO (multiple input multiple output) and beam steering 
antennas [7-12]. For instance, Hong et al. have developed an eight-elements-phased array antenna with beam steering capabilities based on patch antenna design for cellular handsets [7]. The antenna, which is integrated with the cellphone electronic circuits, is capable to steer the radiation pattern over a $60^{\circ}$ range in the $x y$-plane with up to $10.9 \mathrm{dBi}$, depending on the phase applied in each element [7]. Another interesting work on a V-band transceiver module with integrated printed antennas and phased arrays for mm-wave access in 5G mobile networks was realized by Marnat et al. [8]. The proposed structure operates using four phase shifters that can steer the beam to achieve $26.6 \mathrm{dBi}$ gain, aiming outdoor small cell applications. Moreover, Ishfaq et al. have developed a phase-shifted antenna array for outdoor applications, but in this case using massive MIMO to increase the system performance [9]. The 64-element radiating structure provides $24.25 \mathrm{dBi}$ gain and $110^{\circ}$ scanning range [9].

On the other hand, radiators based on different groups of antenna arrays have been exploited for avoiding the use of complex feeding structures $[13,14]$. In those cases, each array is individually and independently excited, with the purpose of proper covering a specific area. However, it is still necessary to use multiple feeding points. Gao et al. [13] introduced a massive MIMO system based on a patch antenna array with 144 feeding ports, which allows to cover $360^{\circ}$. Additionally, by turning different groups of subarrays, it becomes possible to steer the beam to the desired direction [13]. Yang et al. [15] have used the substrate-integrated waveguide (SIW) technology to directly integrate the antenna and transceiver circuit. Their beam steering antenna array is achieved by using digital beamforming (DFB), which provides high flexibility, but it is also complicated in terms of hardware implementation. Each antenna element needs to be associated with a complete $\mathrm{RF}$ chain and a baseband processing unit. All these complex antenna designs, using multiple feeding structures, RF phase shifters and SIW technology, significantly increase the system complexity and cost.

To the best of our knowledge, the majority of publications on mm-wave antennas are based on microstrip antennas [7-14] or SIW technology [15, 16]. Waveguide-based antennas, which are typically used for radar applications, represent a potential and cost-effective solution for $5 \mathrm{G}$ indoor applications, such as corridors, theaters, stadiums, and convention centers, where coverage is not a critical issue. Particularly, the slotted waveguide antenna arrays (SWAAs) present an excellent compromise among cost, complexity, and performance in terms of coverage.

The current work relies on the development of two mmwave waveguide-based antenna arrays for 5G networks: a high-gain omnidirectional antenna array and a dual-band slotted waveguide antenna array. The latter one represents a progress on our previous work recently reported for $5 \mathrm{G}$ networks operating at $28 \mathrm{GHz}$ and $38 \mathrm{GHz}$ [17]. The novel design based on two different slot lengths has been successfully demonstrated by a comparison between the simulated and measured results of the SWAA reflection coefficient and numerical simulations of its radiation pattern. Their applicability is illustrated in Figure 1, which presents two mm-wave indoor scenarios at the INATEL campus:

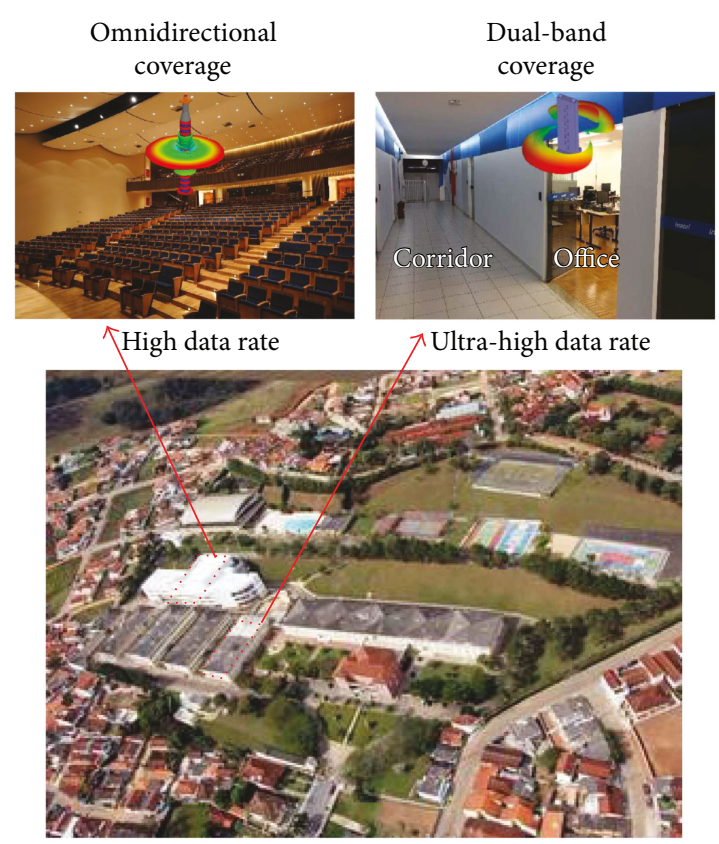

FIgURE 1: Typical scenarios of indoor 5G networks, illustrated at the INATEL campus.

omnidirectional coverage in the INATEL theatre and simultaneous coverage of a corridor and an office by using a dualband antenna array. The two proposed antennas are going to be applied in conjunction with a $5 \mathrm{G}$ modem based on generalized frequency division multiplexing (GFDM), previously developed by our research group in collaboration with the Technical University of Dresden [18], with the purpose of implementing a real $5 \mathrm{G}$ network in our campus.

The manuscript is structured in other three sections. Section 2 reports the development of the high-gain omnidirectional antenna array for the $28 \mathrm{GHz}$ band. The dualband antenna array is presented in Section 3. Finally, Section 4 is regarding the conclusions and future works.

\section{Omnidirectional Ring-Based Slotted Waveguide Antenna Array}

The most commonly used slot antennas are made from slots in the walls of a waveguide. Slotted waveguide antenna arrays (SWAAs) consist of lengths of circular or rectangular waveguide with slots milled into their conducting walls. These slots introduce discontinuities in the conductor and interrupt the current flow in the waveguide wall. On the contrary, the current must flow around the edges of the slots, causing them to act as dipole antennas. Therefore, the entire waveguide structure acts as an array of dipole elements. Their main advantages are simple design and fabrication, low loss, linear polarization, high gain, and power handling. SWAAs are widely used in all kinds of radars, as well as in aircraft applications, since they can be made to conform to the surface on which they are mounted. In this work, we propose to apply this technology for $5 \mathrm{G}$ networks.

Most of SWAAs published in literature are based on rectangular waveguides [19-22]. Particularly, we have recently 


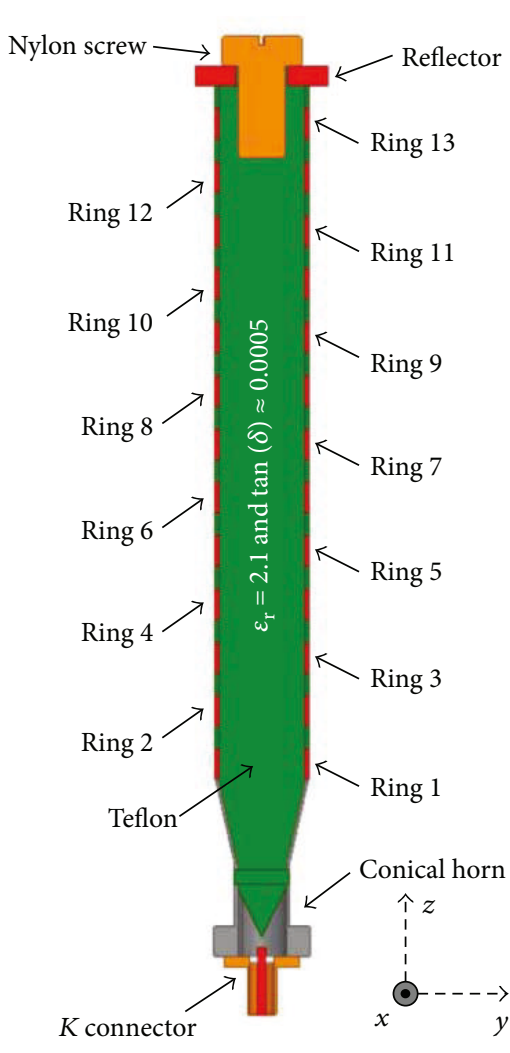

(a) General view

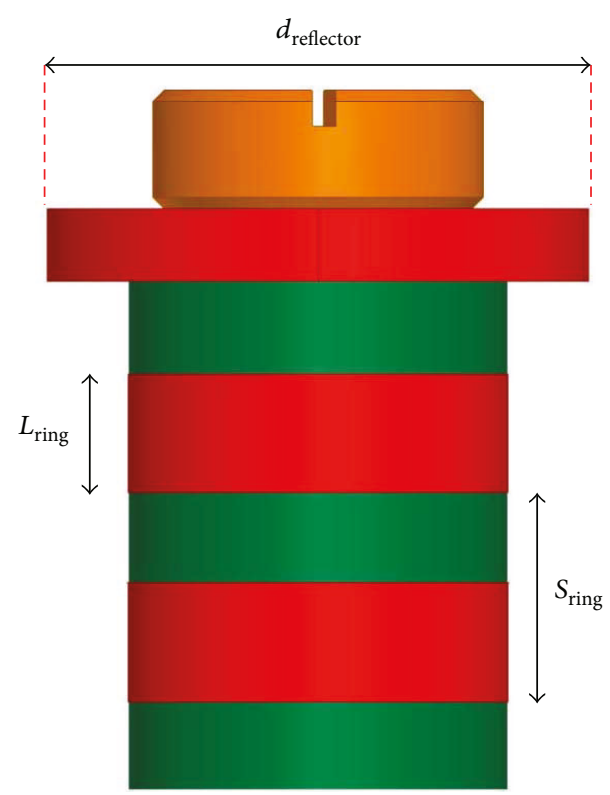

(b) Metallic rings and reflector

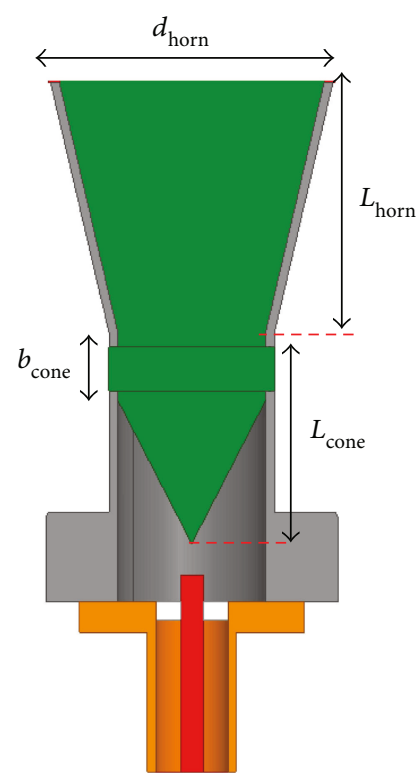

(c) Conical horn

FIGURE 2: Omnidirectional ring-based SWAA: conical horn aperture diameter $\left(d_{\text {horn }}\right)$ and axial length $\left(L_{\text {horn }}\right)$; resonator ring length $\left(L_{\text {ring }}\right)$ and spacing $\left(s_{\text {ring }}\right)$; cone basis and length $\left(b_{\text {cone }}\right)$ and length $\left(L_{\text {cone }}\right)$; reflector diameter $\left(d_{\text {reflector }}\right)$.

reported the concept and development of the first optically controlled reconfigurable antenna for millimeter-wave applications, which is based on a slotted waveguide antenna array and two photoconductive switches [23]. The switches are used to control the slot electrical length in order to enable frequency tunability and radiation pattern reconfiguration through the 28 and $38 \mathrm{GHz}$ frequency bands. Here, we present the development of a high-gain omnidirectional ring-based SWAA based on a circular waveguide, as presented in Figure 2. The waveguide is filled with Teflon-a low-loss polytetrafluoroethylene (PTFE) dielectric material-with electrical permittivity $\varepsilon_{r}=2.1$ and loss tangent tan $\delta=5 \times 10^{-4}$. The physical mechanism behind the radiation in this array is similar to that of omnidirectional leaky wave 


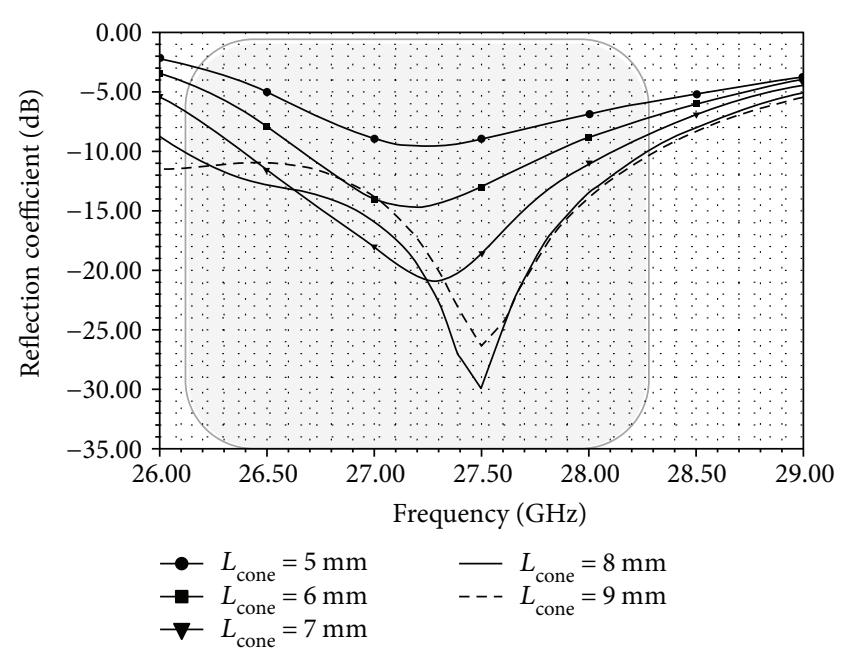

Figure 3: Reflection coefficient of the conical horn as a function of length.

antennas, which are based on a metallic grating loaded with a circular dielectric rod structure $[24,25]$. In our case, we have milled the entire metallic structure, that is, the integration of the waveguide-based periodic metal collar and waveguide feeder, rather than alternating metal and dielectric rings as in [26]. The circular slots act as metallic rings and are equally spaced for guaranteeing a structure circularly symmetric. We have used a metallic reflector and a nylon screw for closing the radiating structure.

The periodically structured rings into a dielectric rod create a perturbation in the guided electromagnetic waves, in such a way, the proposed ring based behaves as a leaky wave antenna, in which the energy is gradually radiated through the slots to the air, as it propagates into the cylindrical dielectric. The omnidirectional pattern in the $x y$-plane is ensured by adjusting the resonator ring spacing $\left(S_{\text {ring }}\right)$. The circularly symmetrical radiating structure is excited by rotationally symmetrical fields, such as $\mathrm{TM}_{01}$ or $\mathrm{TE}_{01}$ modes, with the purpose of providing an omnidirectional radiation pattern [27]. A conical horn fed by a longitudinal $K$-connector ensures the desired $\mathrm{TM}_{01}$-guided mode. Its main dimensions are the axial length $\left(L_{\text {horn }}\right)$ and aperture diameter $\left(d_{\text {horn }}\right)$. The antenna directivity depends on the aperture diameter and operational wavelength $(\lambda)$, accordingly to the following equation [28]:

$$
D=\varepsilon_{\text {ap }}\left(\frac{d_{\text {horn }} \pi}{\lambda}\right)^{2}
$$

where $\varepsilon_{\text {ap }}$ is aperture efficiency. The ring-based SWAA beam direction angle $(\theta)$ depends on the ring length $\left(L_{\text {ring }}\right)$ and spacing $\left(S_{\text {ring }}\right)$. It can be calculated by [27]

$$
\begin{aligned}
& \cos \theta=\left(\frac{\beta}{k_{0}}-\frac{2 \pi}{k_{0} s_{\text {ring }}}\right), \\
& L_{\text {ring }} \approx 0.6 s_{\text {ring }},
\end{aligned}
$$

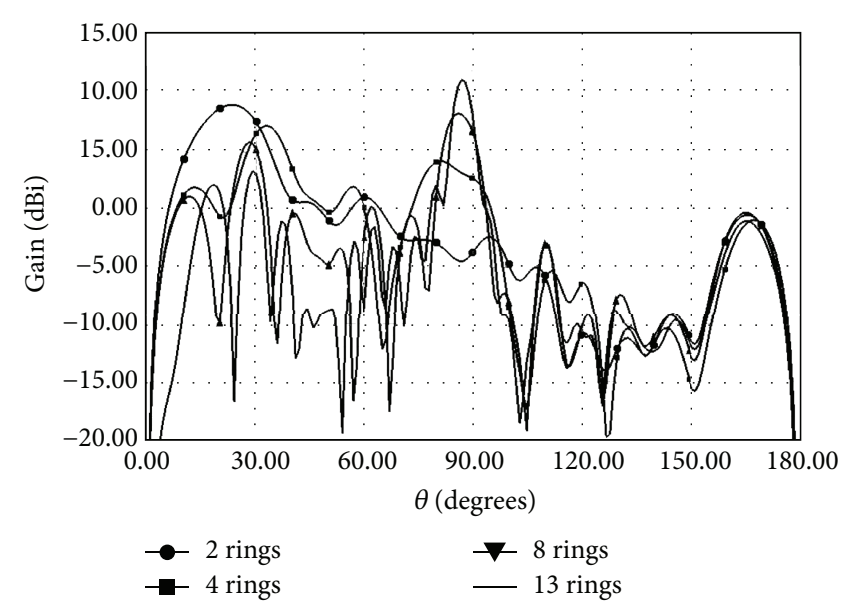

FIGURE 4: Radiation pattern analysis as a function of the number of rings.

TABLE 1: Analysis of the number of rings of the omnidirectional ring-based SWAA.

\begin{tabular}{lcc}
\hline Number of rings & Gain $(\mathrm{dBi})$ & SLL $(\mathrm{dB})$ \\
\hline 2 & 8.93 & -8.85 \\
4 & 7.25 & -3.30 \\
6 & 6.54 & -0.47 \\
8 & 8.55 & -2.92 \\
10 & 9.63 & -5.15 \\
13 & 10.84 & -7.24 \\
14 & 10.10 & -7.96 \\
\hline
\end{tabular}

where $\beta$ is the propagation constant of the $\mathrm{TM}_{01}$ mode propagating along the circular dielectric rod waveguide and $k_{0}$ is the free-space wavenumber.

The conical horn antenna dimensions have been defined as $d_{\text {horn }}=15 \mathrm{~mm}$ and $L_{\text {horn }}=9.92 \mathrm{~mm}$ for providing a gain of $10 \mathrm{dBi}$ and operation from 24.0 to $33.0 \mathrm{GHz}$. It has been filled with PTFE in order to mechanically support the ring-based SWAA. Moreover, a tapered transition with length $L_{\text {cone }}$ has been inserted between PTFE and air, with the purpose of improving the antenna impedance matching. Figure 3 presents the numerical analysis of the SWAA reflection coefficient $\left(S_{11}\right)$ as a function of $L_{\text {cone }}$. The best result has been obtained for $L_{\text {cone }}=8 \mathrm{~mm}$, which implies in a $S_{11}$ dip of $-30 \mathrm{~dB}$ at $27.5 \mathrm{GHz}$.

The ring-based SWAA radiation pattern strongly depends on the ring dimensions. In accordance to (2), the ring length and spacing should be equal to 10.71 and $6.43 \mathrm{~mm}$, respectively, in order to provide broadside radiation, that is, the antenna main lobe normal to its axis $\left(\theta=90^{\circ}\right)$. Figure 4 reports a numerical analysis of the ring-based SWAA radiation pattern as a function of the number of rings, whereas Table 1 summarizes its most important electromagnetic parameters. The main requirement is providing broadside radiation at a low side lobe level (SLL). Thirteen elements represent the best compromise, that is, $G=10.84 \mathrm{dBi}$ and SLL $=-7.24 \mathrm{~dB}$. A further increase in the number of elements implies in gain reduction, as proved for fourteen elements in Table 1. 
TABLe 2: Analysis of the reflector diameter of the omnidirectional ring-based SWAA.

\begin{tabular}{lcc}
\hline$d_{\text {reflector }}(\mathrm{mm})$ & Gain $(\mathrm{dBi})$ & SLL $(\mathrm{dB})$ \\
\hline 0 & 10.84 & -7.24 \\
15 & 10.85 & -10.58 \\
20 & 11.48 & -11.22 \\
23 & 12.25 & -12.60 \\
26 & 11.31 & -11.84 \\
30 & 11.34 & -11.32 \\
\hline
\end{tabular}

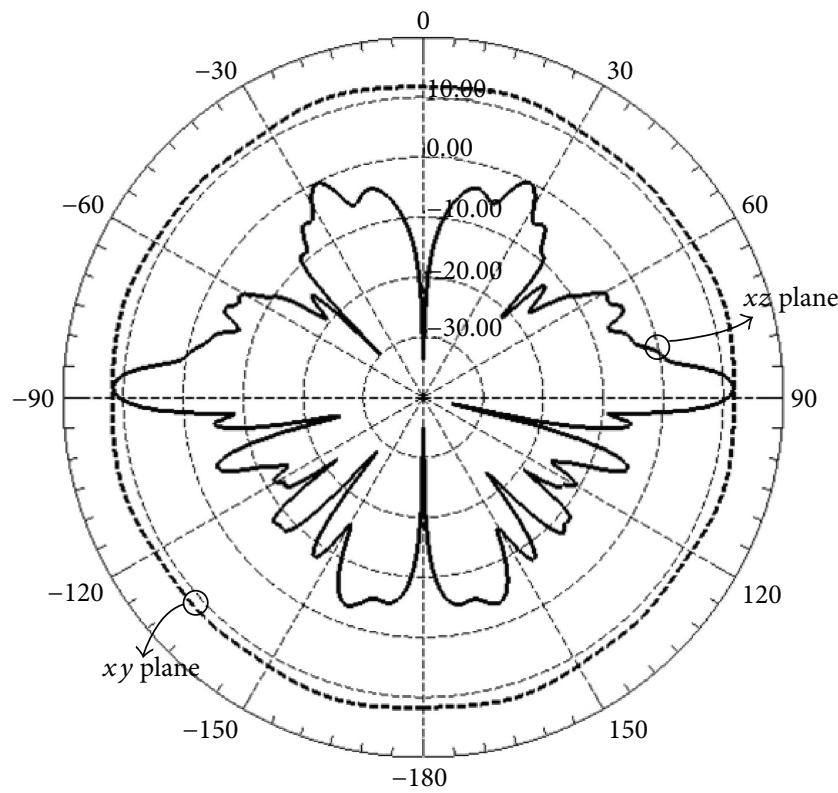

Figure 5: Radiation pattern, in $\mathrm{dBi}$, of the omnidirectional ringbased SWAA at $28 \mathrm{GHz}$.

The metallic reflector width has been defined as $1.8 \mathrm{~mm}$, which corresponds to one-quarter of the guided wavelength, for minimizing the side lobe level. Its diameter has been numerically evaluated by using ANSYS HFSS, as displayed in Table 2 . The diameter $d_{\text {reflector }}=23 \mathrm{~mm}$ implies in the best side lobe level $(-12.60 \mathrm{~dB})$ and gain $(12.24 \mathrm{dBi})$ results.

Figure 5 presents the two-dimensional radiation patterns of the omnidirectional ring-based SWAA at 28.0 GHz. The gain variation in $x y$-plane is only $0.88 \mathrm{~dB}$, making up an omnidirectional pattern with $12.25 \mathrm{dBi}$ remarkable gain, which is ten times higher than that of a conventional halfwave dipole antenna. This gain enhancement is very interesting to handle with high losses of $5 \mathrm{G}$ networks operating in the mm-wave range.

Figure 6 reports a photograph of the omnidirectional ring-based SWAA prototype. We have realized product engineering in order to make our numerical model (Figure 6(a)) manufacturable. The required adaptations have not significantly changed the SWAA electromagnetic properties. Figure 7 presents the experimental characterization of ringbased SWAA, as well as comparisons between the numerical and experimental results. Its reflection coefficient has been measured using a Keysight FieldFox Microwave Analyzer

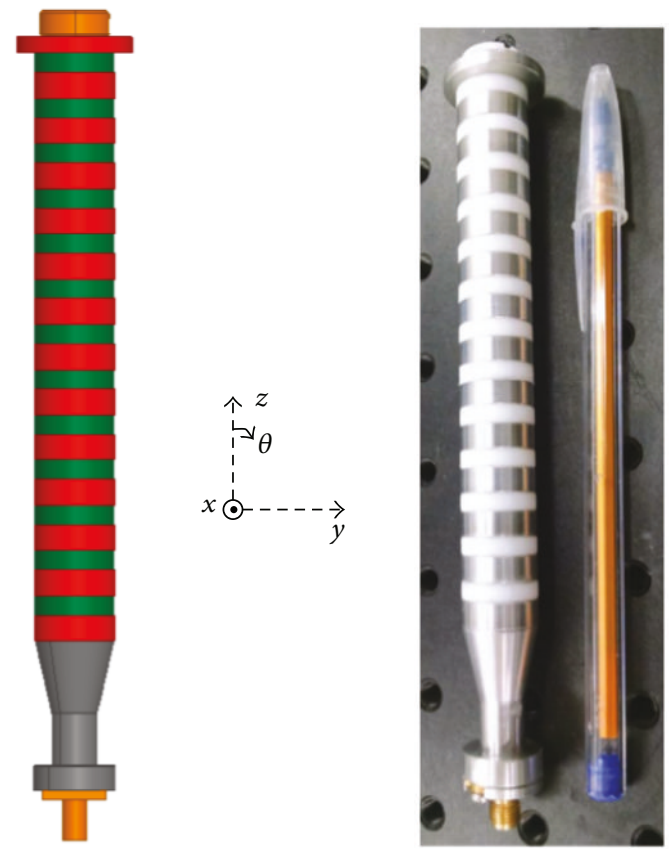

(a) Numerical model

(b) Prototype

FIgURE 6: Omnidirectional ring-based SWAA.

N9952A. A maximum level of $-10 \mathrm{~dB}$ had been assumed to define the antenna bandwidth. A good qualitative agreement is observed; nonetheless, the measured bandwidth is wider than that of the HFSS simulations. The proposed ringbased SWAA provides a bandwidth of approximately $7.7 \%$ from 26.85 to $29 \mathrm{GHz}$. Its measured radiation pattern in the azimuth plane ( $x y$-plane) at $28 \mathrm{GHz}$, reported in Figure $7(\mathrm{~b})$, is in excellent agreement with the HFSS simulation. The SWAA measured gain was $12.25 \mathrm{dBi}$ for $28 \mathrm{GHz}$, which is in agreement with the numerical prediction.

Figures $7(\mathrm{c})-7(\mathrm{e})$ display the radiation pattern in the elevation plane ( $x z$-plane) for five different frequencies through the antenna bandwidth. As expected for leaky wave antennas, it is observed a beam pointing swept of five degrees from the bandwidth lowest frequency to the highest one, either in the simulations (Figure $7(\mathrm{~d})$ ) and measurements (Figure 7(e)). It is important to highlight that the half-power beamwidth is kept and the gain variation is lower than $3 \mathrm{~dB}$ over the antenna entire bandwidth, making feasible to apply it to transmit/receive broadband wireless signals with up to $2.15 \mathrm{GHz}$ bandwidth. As a conclusion, the proposed highgain ring-based SWAA might be considered potential for omnidirectional coverage in mm-wave applications, such as shopping malls, theatres, convention centers, and stadiums.

\section{Dual-Band Slotted Waveguide Antenna Array}

This section is regarding the development of a dual-band sectorial slotted waveguide antenna array for ultrahigh communication indoor mm-wave $5 \mathrm{G}$ networks. Conventional SWAAs are typically narrow and single band [19, 21, 22]. Their bandwidth is determined by the waveguide structure and resonant properties of the individual slots. One possible 


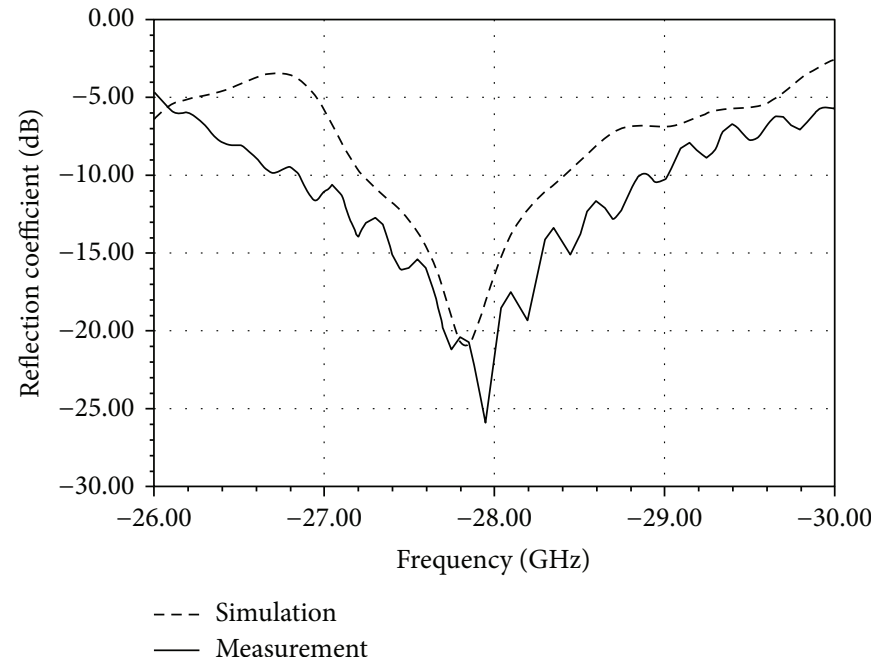

(a) Reflection coefficient

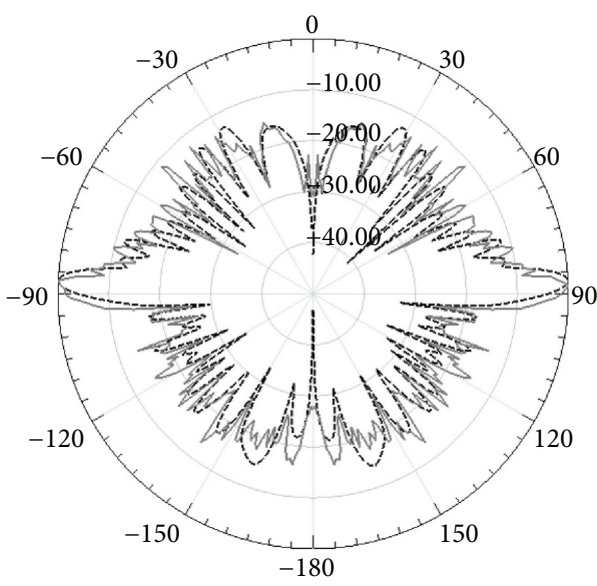

(c) Radiation pattern, in $\mathrm{dBi}$, at $28 \mathrm{GHz}$ in the $x z$-plane

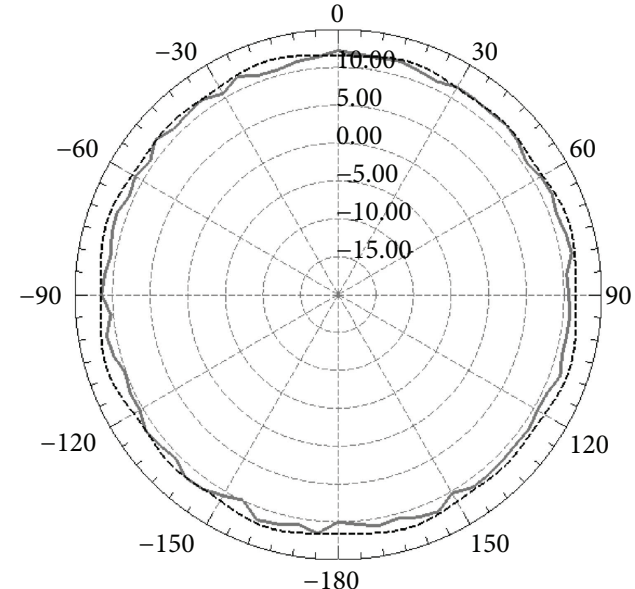

(b) Radiation pattern, in $\mathrm{dBi}$, at $28.0 \mathrm{GHz}$ in the $x y$ -

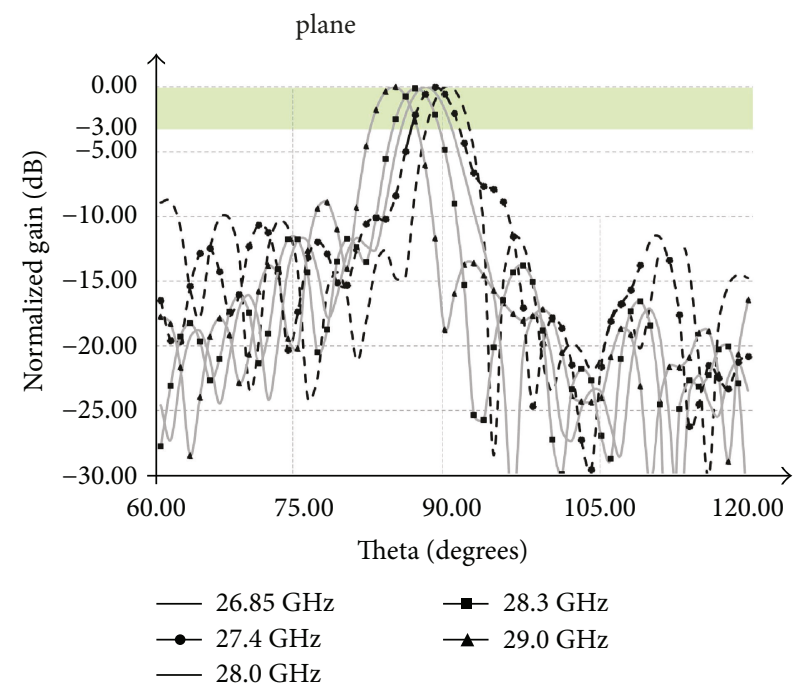

(d) Beam pointing simulation in the $x z$-plane

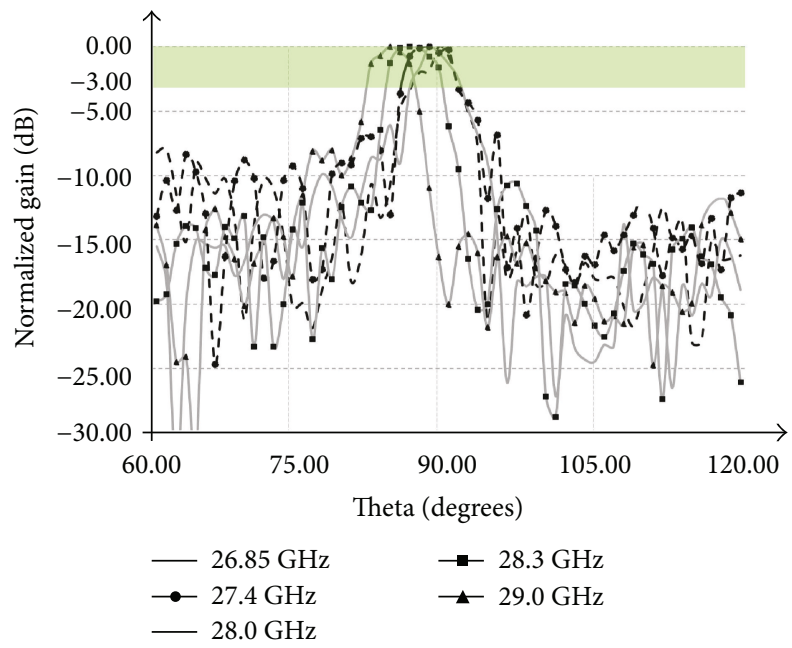

(e) Beam pointing measurement in the $x z$-plane

FIGURE 7: Characterization of the omnidirectional ring-based SWAA: simulation (dashed lines); measurements (continuous lines). 


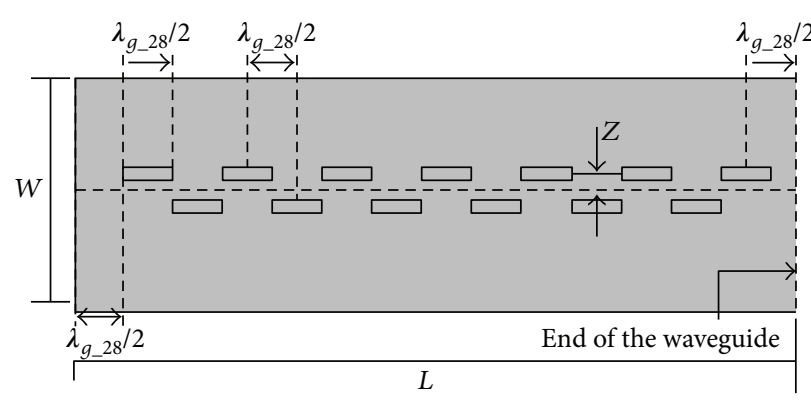

(a) Slots for $28 \mathrm{GHz}$

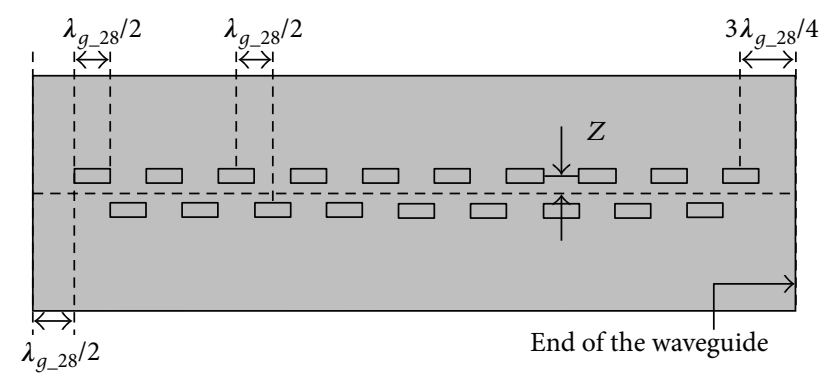

(b) Slots for $38 \mathrm{GHz}$

FIgURe 8: Dual-band SWAA model.

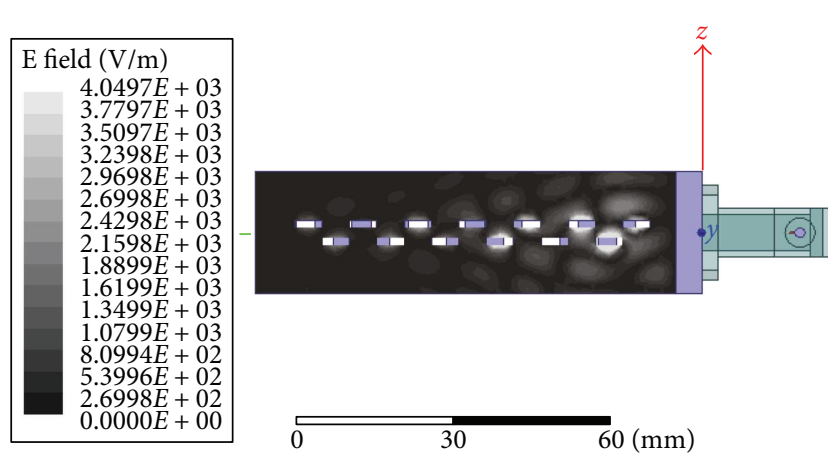

(a) $28 \mathrm{GHz}$
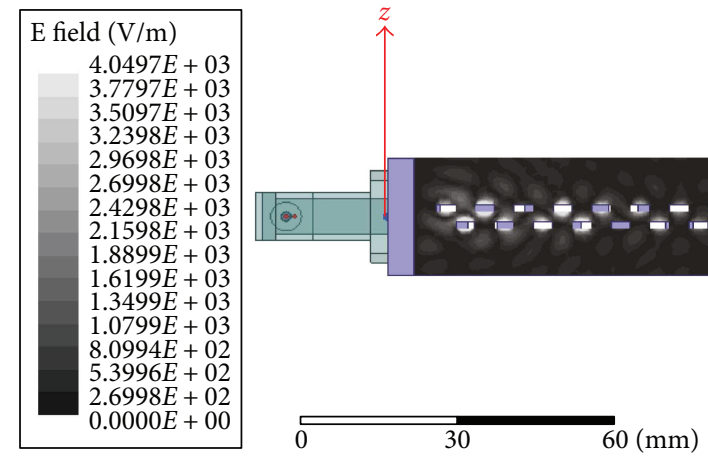

(b) $38 \mathrm{GHz}$

FIGURE 9: Electric field distribution on the slots.

strategy to enhance the SWAA bandwidth is making their slots wider. We have recently reported multiband SWAAs based on a new approach $[17,22,29]$, which relies on using two different slot lengths and separations in order to provide a wider bandwidth, by means of using slots with distinct electrical lengths and, consequently, different resonances. The current SWAA model exploits the same technique for increasing the antenna bandwidth, but now milling two groups of slots with different lengths into the waveguide opposite broad walls, as proposed in [17] and described in Figure 8 . The present manuscript represents progress of our previous work [17], since it presents numerical simulations of the electrical field and measurements of the antenna radiation pattern.

The SWAA design is typically based on the design procedures published by Elliot [19]. The slot length and interslot center-to-center distance are approximately onehalf of the guided wavelength. On the other hand, the distance between the last slot center and structure end is $(2 p+1)(\lambda g / 4)$, in which $p$ is a positive integer number, in order to guarantee the slots will be placed in the electric field maximum points. The slots must be placed at the antinode locations, where the electric field reaches its maximum, because the waveguide hosts a standing wave. SWAA designs commonly do not take into account mutual coupling between slots. Since mutual coupling and waveguide thickness have a significant influence on the edge-slot admittances, an accurate analysis of the antenna array should take their effects into account. ANSYS HFSS can model these both effects, as well as those due to coaxial transitions and flanges. In this way, the traditional empirical trial-and-error method is replaced by a computational evaluation in order to proper numerically optimized all the SWAA design parameters for enhancing its performance.

The proposed SWAA has been idealized using a standing wave structure (the waveguide end is short circuited), in which the wave travels inside the waveguide bouncing back and forth between the ends of the antenna structure. This technique takes advantage from the reflected portion of the energy, thus increasing the radiation efficiency, compared to the traveling wave antenna SWAAs. It has two slot groups milled on each waveguide broadside face, with the purpose of allowing simultaneous operation over 28 and $38 \mathrm{GHz}$ frequency bands. Figure 8(a) presents the side view of the slots designed for $28 \mathrm{GHz}$ and its dimensions. The slots for $38 \mathrm{GHz}$ have been milled in the opposite waveguide face, as illustrated in Figure 8(b). We have defined the distance between the last slot center and the waveguide end as $\left(3 \times \lambda_{g_{-} 38}\right) / 4$ and $\lambda_{g_{\_} 28} / 2$ for 38 and $28 \mathrm{GHz}$, respectively. Therefore, the condition for guaranteeing the slots will be placed in the electric field maxima $((2 p+1)(\lambda g / 4))$ has only been respected for $38 \mathrm{GHz}$, which was our priority, because its free-space attenuation is higher than that of the $28 \mathrm{GHz}$ band. In any case, this feature does not imply in any degradation on the antenna performance, since the energy that arrives to the waveguide end is very weak, since it is gradually radiated across the slots; thus, the reflected energy is not prejudicial. Figure 9 presents the 


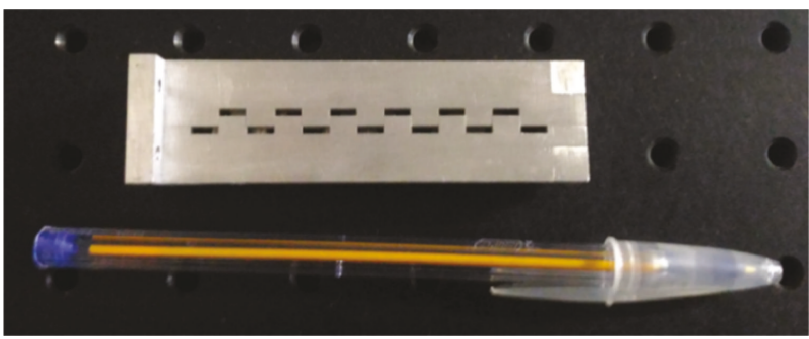

(a) Slots for $28 \mathrm{GHz}$

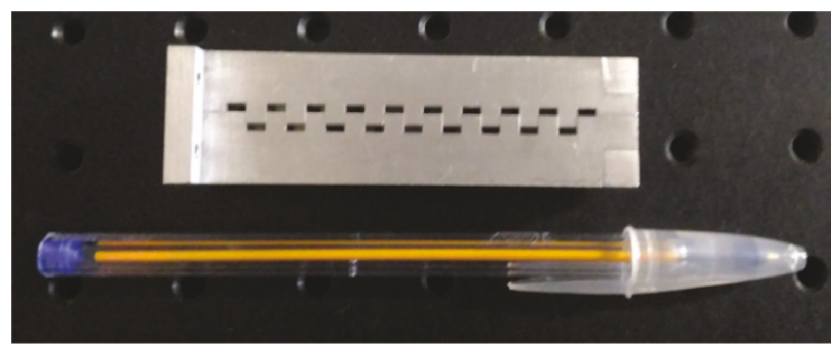

(b) Slots for $38 \mathrm{GHz}$

FIGURE 10: Dual-band slotted waveguide antenna array.

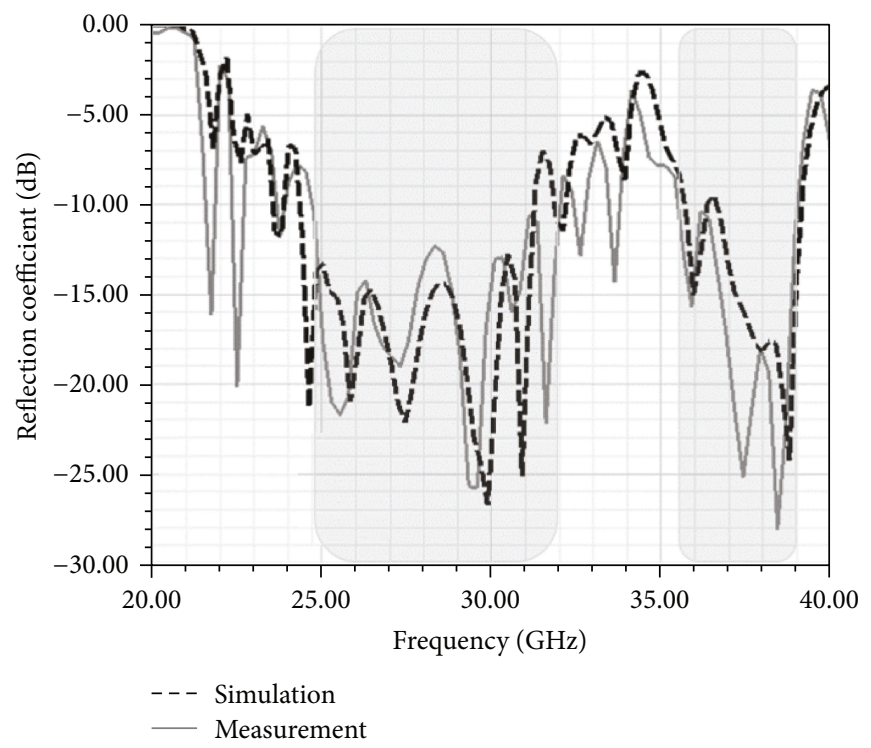

FIGURE 11: Reflection coefficient of the proposed dual-band SWAA.

field distribution in the structure proving the slot excitation. The guided wavelengths for 28 and $38 \mathrm{GHz}$ are $\lambda_{g \_28}=10.7 \mathrm{~mm}$ and $\lambda_{g \_38}=7.4 \mathrm{~mm}$, respectively; thus, the dual-band SWAA final dimensions are $Z=1.77 \mathrm{~mm}$, $W=24.1 \mathrm{~mm}$, and $L=87.4 \mathrm{~mm}$. The difference between the groups of slots is only their lengths, since their spacing, width, and separation to the centerline are the same.

The dual-band SWAA has been made of aluminum, as the previous one. The feeding structure has been designed to easily be connected to a standard coax/WR28 $(7.11 \times 3.56 \mathrm{~mm})$ waveguide transition, which had also been considered in the numerical simulations. Figure 10 reports photographs of the two waveguide opposite faces with the slots for 28 and $38 \mathrm{GHz}$. The first antenna characterization step was aimed to validate the antenna operation, by means of measuring its reflection coefficient, presented in Figure 11. This SWAA provides two wide bandwidths: the first one of $26.36 \%$ from 24.7 to $32.2 \mathrm{GHz}$; the second one of $9.78 \%$ from 35.5 to $39.15 \mathrm{GHz}$.

Figure 12 reports an excellent agreement between numerical simulations and experimental results of the dual-band SWAA radiation pattern in the azimuth plane at 28 and $38 \mathrm{GHz}$. Its slots have been arranged for providing broadside radiation and vertical polarization for both designed frequency bands, as required in cellular networks. Particularly for the $38 \mathrm{GHz}$ band, the signal can also be radiated through both antenna sides, since it represents the smaller wavelength that naturally can pass through the slots designed for the longer wavelength, designed for the $28 \mathrm{GHz}$ band. On the other hand, the lower frequency band concentrates its radiation only in one side. The proposed dual-band SWAA provides a gain of 12.6 and $15.6 \mathrm{dBi}$ for 28 and $38 \mathrm{GHz}$, respectively. The gain difference between the two operational bands is due to the larger number of slots of the $38 \mathrm{GHz}$ band, since its wavelength is smaller than that of the $28 \mathrm{GHz}$ band. Furthermore, our design does not allow radiation in the antenna perpendicular axis for both frequency bands. As a result of the propagation through the waveguide, the main lobe points to the same angle for both frequencies, but in opposite faces, namely, $105^{\circ}$ for $28 \mathrm{GHz}$ and $255^{\circ}$ for $38 \mathrm{GHz}$. This characteristic occurs due to the amplitude distribution on the slots. As the signal radiates through them, the remaining energy inside the waveguide has its amplitude decreased, exciting each slot with different amplitude values, as demonstrated in the electrical field plots from Figure 9. The first slot receives the full amplitude signal 


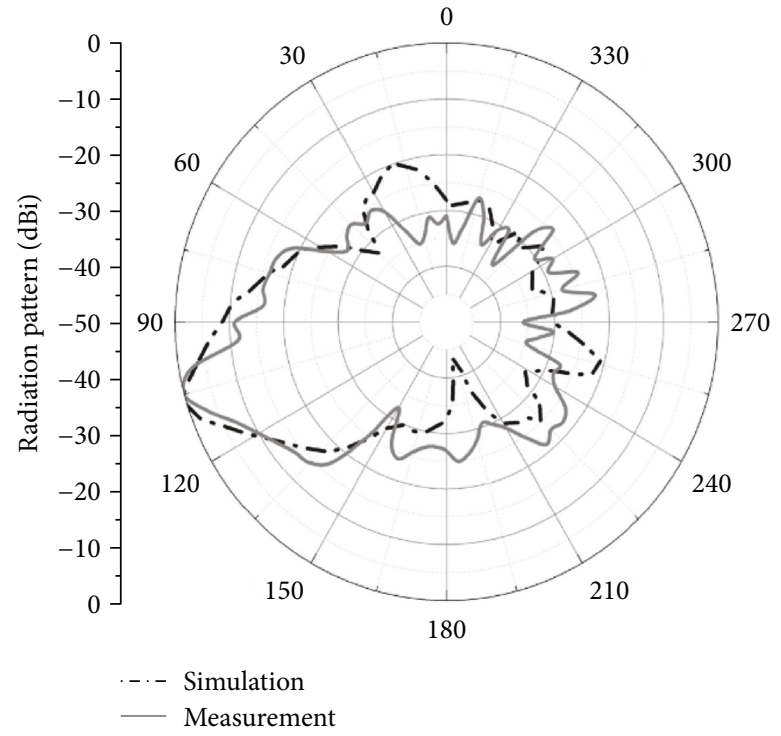

(a) $28 \mathrm{GHz}$

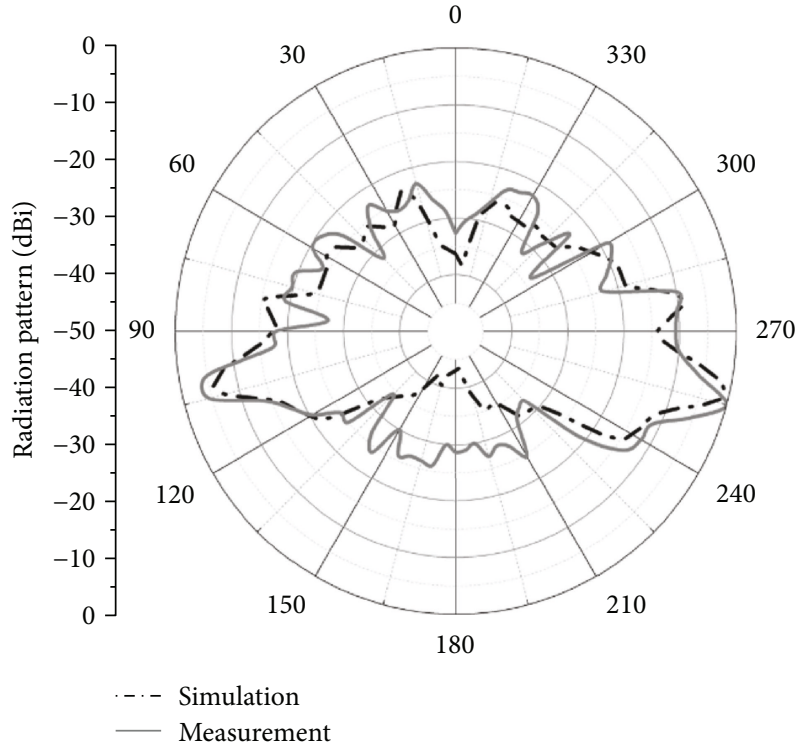

(b) $38 \mathrm{GHz}$

FIGURE 12: Dual-band SWAA radiation pattern in the azimuth plane.

from the feeding point, shifting the radiation pattern toward itself; this means, $105^{\circ}$ and $255^{\circ}$ for 28 and $38 \mathrm{GHz}$, respectively.

As a conclusion, the proposed dual-band SWAA can be applied to adaptive 5G networks in two different ways as a function of the radio mobile environment and customer quality of service: using two different bands independently; applying carrier aggregation utilizing 28 and $38 \mathrm{GHz}$ bands at the same time. As proposed in Figure 1, the use of two distinct frequency bands can simultaneously enable high data rate in a corridor, where people are passing through, and ultrahigh data rates for users inside offices, which naturally demands higher throughput.

\section{Conclusions}

We have successfully proposed and developed two innovative and novel slotted waveguide antenna arrays for $\mathrm{mm}$ wave 5G networks, namely, $12 \mathrm{dBi}$ gain omnidirectional antenna array and dual-band antenna array. The first produced SWAA operates in the $28 \mathrm{GHz}$ band, whereas the latter one enables dual-band operation in the 28 and $38 \mathrm{GHz}$ bands, which could allow the use of carrier aggregation in $5 \mathrm{G}$ networks. The array bandwidth varies from 7.9 to $26.36 \%$, as a result of a novel approach recently proposed by authors to enhance SWAA bandwidth [22, 29]. Specific radiation patterns have been demonstrated with gains up to $15.6 \mathrm{dBi}$.

The array applicability has been illustrated by proposing two mm-wave scenarios in our campus: omnidirectional coverage at the INATEL theatre and simultaneous indoor coverage of a corridor and an office using a dual-band antenna array. Future works are regarding the implementation of the two proposed 5G scenarios in our campus using the two produced slotted waveguide antenna arrays and a
GFDM (Generalized Frequency Division Multiplexing) modem, previously developed by our research group in collaboration with the Dresden Technical University from Germany $[18,30]$.

\section{Conflicts of Interest}

The authors declare that they have no conflicts of interest.

\section{Acknowledgments}

This work was partially supported by FINEP/Funttel Grant no. 01.14.0231.00, under the Radio Communications Reference Centre (CRR) project of the National Institute of Telecommunications (INATEL), Brazil. The authors also thank the financial support from $\mathrm{CNPq}$, CAPES, MCTI, and FAPEMIG and technical support from Matheus Borsato-INATEL, Anritsu, Keysight, and ESSS-ANSYS.

\section{References}

[1] T. S. Rappaport, S. Sun, R. Mayzus et al., "Millimeter wave mobile communications for $5 \mathrm{G}$ cellular: it will work!," IEEE Access, vol. 1, pp. 335-349, 2013.

[2] A. I. Sulyman, A. Alwarafy, G. R. MacCartney, T. S. Rappaport, and A. Alsanie, "Directional radio propagation path loss models for millimeter-wave wireless networks in the 28-, 60-, and 73-GHz bands," IEEE Transactions on Wireless Communications, vol. 15, no. 10, pp. 6939-6947, 2016.

[3] W. Chin, Z. Fan, and R. Haines, "Emerging technologies and research challenges for $5 \mathrm{G}$ wireless networks," IEEE Wireless Communications, vol. 21, no. 2, pp. 106-112, 2014.

[4] C. X. Wang, F. Haider, X. Gao et al., "Cellular architecture and key technologies for $5 \mathrm{G}$ wireless communication networks," 
IEEE Communications Magazine, vol. 52, no. 2, pp. 122-130, 2014.

[5] G. R. MacCartney Jr., S. Sun, T. S. Rappaport et al., "Millimeter wave wireless communications: new results for rural connectivity," in Proceedings of the 5th Workshop on All Things Cellular Operations, Applications and Challenges - ATC '16, pp. 31-36, New York City, NY, USA, October 2016, ACM MobiCom.

[6] "[5G] spectrum recommendations," August 2015, http:// www.5gamericas.org.

[7] W. Hong, K. H. Baek, and S. Ko, "Millimeter-wave 5G antennas for smartphones: overview and experimental demonstration," IEEE Transactions on Antennas and Propagation, vol. 65, no. 12, pp. 6250-6261, 2017.

[8] L. Marnat, L. Dussopt, V. Puyal et al., "V-band transceiver modules with integrated antennas and phased arrays for mmWave access in 5G mobile networks," in 2017 11th European Conference on Antennas and Propagation (EUCAP), pp. 2786-2790, Paris, France, 2017.

[9] M. K. Ishfaq, T. A. Rahman, Y. Yamada, and K. Sakakibara, " $8 \times 8$ phased series fed patch antenna array at $28 \mathrm{GHz}$ for $5 \mathrm{G}$ mobile base station antennas," in 2017 IEEE-APS Topical Conference on Antennas and Propagation in Wireless Communications (APWC), pp. 160-162, Verona, Italy, 2017.

[10] Y. W. Hsu, T. C. Huang, H. S. Lin, and Y. C. Lin, "Dual-polarized Quasi Yagi-Uda antennas with EndFire radiation for millimeter-wave MIMO terminals," IEEE Transactions on Antennas and Propagation, vol. 65, no. 12, pp. 6282-6289, 2017.

[11] M. S. Sharawi, M. Ikram, and A. Shamim, "A two concentric slot loop based connected array MIMO antenna system for 4G/5G terminals," IEEE Transactions on Antennas and Propagation, vol. 65, no. 12, pp. 6679-6686, 2017.

[12] S. X. Ta, H. Choo, and I. Park, "Broadband printed-dipole antenna and its arrays for 5G applications," IEEE Antennas and Wireless Propagation Letters, vol. 16, pp. 2183-2186, 2017.

[13] Y. Gao, R. Ma, Y. Wang, Q. Zhang, and C. Parini, "Stacked patch antenna with dual-polarization and low mutual coupling for massive MIMO," IEEE Transactions on Antennas and Propagation, vol. 64, no. 10, pp. 4544-4549, 2016.

[14] W. Hong, Z. H. Jiang, C. Yu et al., "Multibeam antenna technologies for $5 \mathrm{G}$ wireless communications," IEEE Transactions on Antennas and Propagation, vol. 65, no. 12, pp. 6231-6249, 2017.

[15] B. Yang, Z. Yu, Y. Dong, J. Zhou, and W. Hong, "Compact tapered slot antenna array for 5G millimeter-wave massive MIMO systems," IEEE Transactions on Antennas and Propagation, vol. 65, no. 12, pp. 6721-6727, 2017.

[16] S. J. Park, D. H. Shin, and S. O. Park, "Low side-lobe substrateintegrated-waveguide antenna array using broadband unequal feeding network for millimeter-wave handset device," IEEE Transactions on Antennas and Propagation, vol. 64, no. 3, pp. 923-932, 2016.

[17] I. F. da Costa, S. Arismar Cerqueira Jr., and D. H. Spadoti, "Dual-band slotted waveguide antenna array for adaptive mm-wave 5G networks," in 2017 11th European Conference on Antennas and Propagation (EUCAP), pp. 1322-1325, Paris, France, 2017.
[18] N. Michailow, M. Matthe, I. S. Gaspar et al., "Generalized frequency division multiplexing for 5 th generation cellular networks," IEEE Transactions on Communications, vol. 62, no. 9, pp. 3045-3061, 2014.

[19] R. Elliott, "An improved design procedure for small arrays of shunt slots," IEEE Transactions on Antennas and Propagation, vol. 31, no. 1, pp. 48-53, 1983.

[20] S. Liao, J. Wang, Y. Chen et al., "Synthesis, simulation and experiment of unequally spaced resonant slotted-waveguide antenna arrays based on the infinite wavelength propagation property of composite right/left-handed waveguide," IEEE Transactions on Antennas and Propagation, vol. 60, no. 7, pp. 3182-3194, 2012.

[21] S. Liao, P. Chen, P. Wu, K. M. Shum, and Q. Xue, "Substrateintegrated waveguide-based $60-\mathrm{GHz}$ resonant slotted waveguide arrays with wide impedance bandwidth and high gain," IEEE Transactions on Antennas and Propagation, vol. 63, no. 7, pp. 2922-2931, July.

[22] S. Arismar Cerqueira Jr., I. F. da Costa, S. Pinna et al., "A novel dual-polarization and dual-band slotted waveguide antenna array for dual-use radars," in 2016 10th European Conference on Antennas and Propagation (EuCAP), pp. 1-4, Davos, Switzerland, 2016.

[23] I. F. da Costa, A. Cerqueira S., D. H. Spadoti, L. G. da Silva, J. A. J. Ribeiro, and S. E. Barbin, "Optically controlled reconfigurable antenna array for mm-wave applications," IEEE Antennas and Wireless Propagation Letters, vol. 16, pp. 2142-2145, 2017.

[24] S. Xu, J. Min, S.-T. Peng, and F. K. Schwering, "A millimeterwave omnidirectional circular dielectric rod grating antenna," IEEE Transactions on Antennas and Propagation, vol. 39, no. 7, pp. 883-891, 1991.

[25] $\mathrm{S} . \mathrm{Xu}$ and $\mathrm{X}$. Wu, "A millimeter-wave omnidirectional dielectric rod metallic grating antenna," IEEE Transactions on Antennas and Propagation, vol. 44, no. 1, pp. 74-79, 1996.

[26] T. Iwasaki, T. Ikeda, and T. Kuki, "Millimeter-wave beamswitchable circular dielectric rod antenna using a periodic metal collar," in Proceedings of ISAP, 2007, pp. 121-124, Niigata, Japan, 2007.

[27] T. Iwasaki and T. Ikeda, "A study on beam-switching dielectric-rod antenna using periodic metal plate," Tech. Rep. AP2004-232, IEICE, Japan, 2005.

[28] C. Balanis, Antenna Theory: Analysis and Design, John Wiley \& Sons, New York, NY, USA, 2012.

[29] I. F. da Costa, A. C. Sodré, L. G. Silva, D. H. Spadoti, and A. Bogoni, "Tri-band slotted waveguide antenna array for millimetric-waves applications," in The 8th European Conference on Antennas and Propagation (EuCAP 2014), pp. 2052 2054, The Hague, Netherlands, April 2014.

[30] M. Matthe, L. L. Mendes, N. Michailow, D. Zhang, and G. Fettweis, "Widely linear estimation for space-time-coded GFDM in low-latency applications," IEEE Transactions on Communications, vol. 63, no. 11, pp. 4501-4509, 2015. 


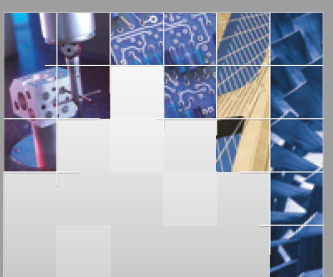

\section{Enfincering}
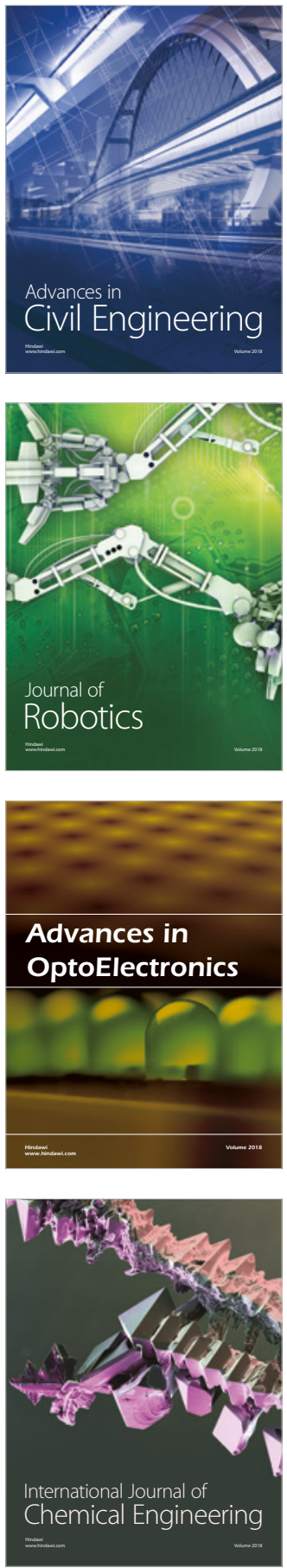

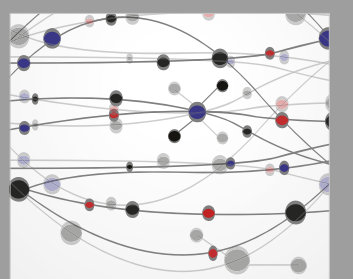

\section{Rotating \\ Machinery}

The Scientific World Journal

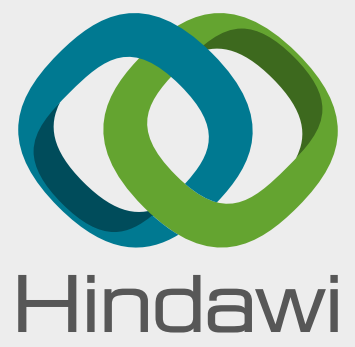

Submit your manuscripts at

www.hindawi.com
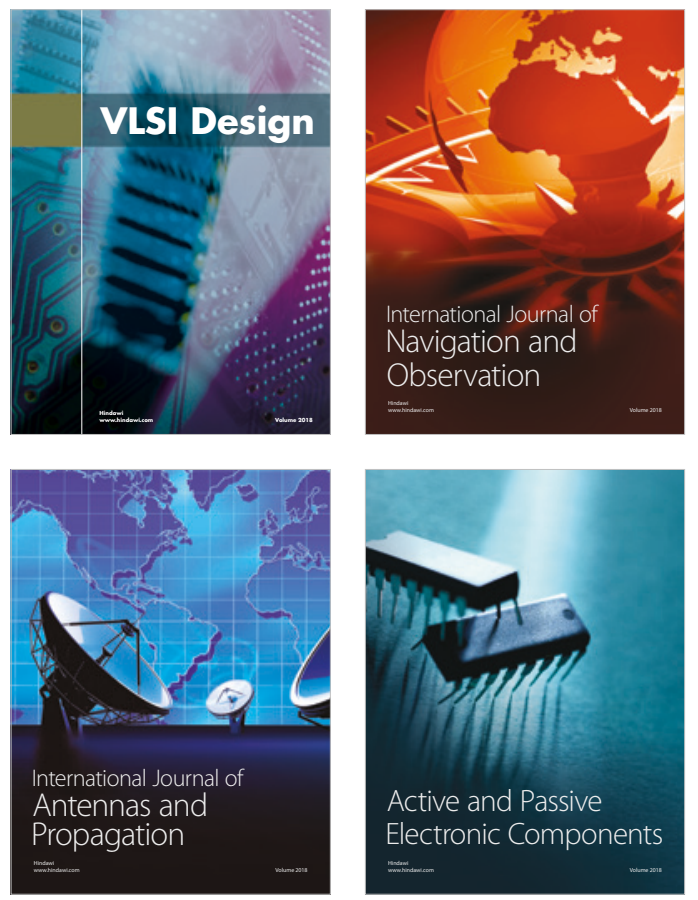
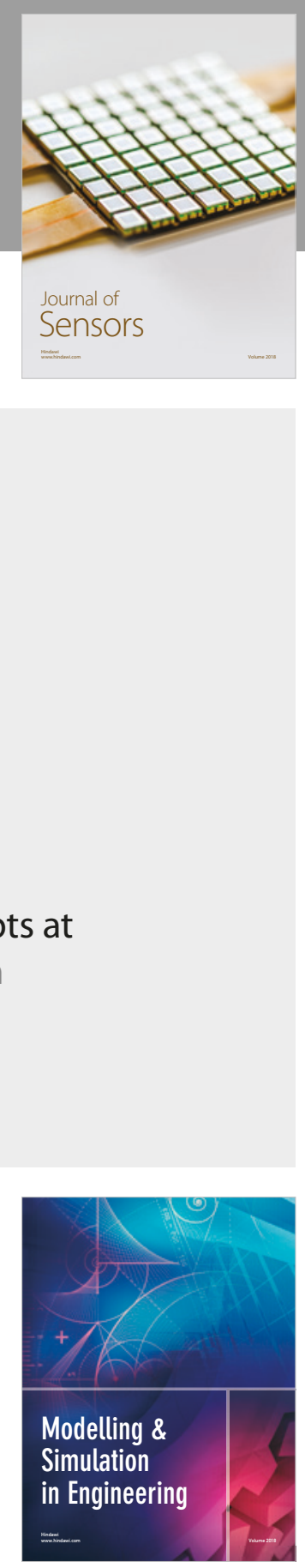

\section{Advances \\ Multimedia}
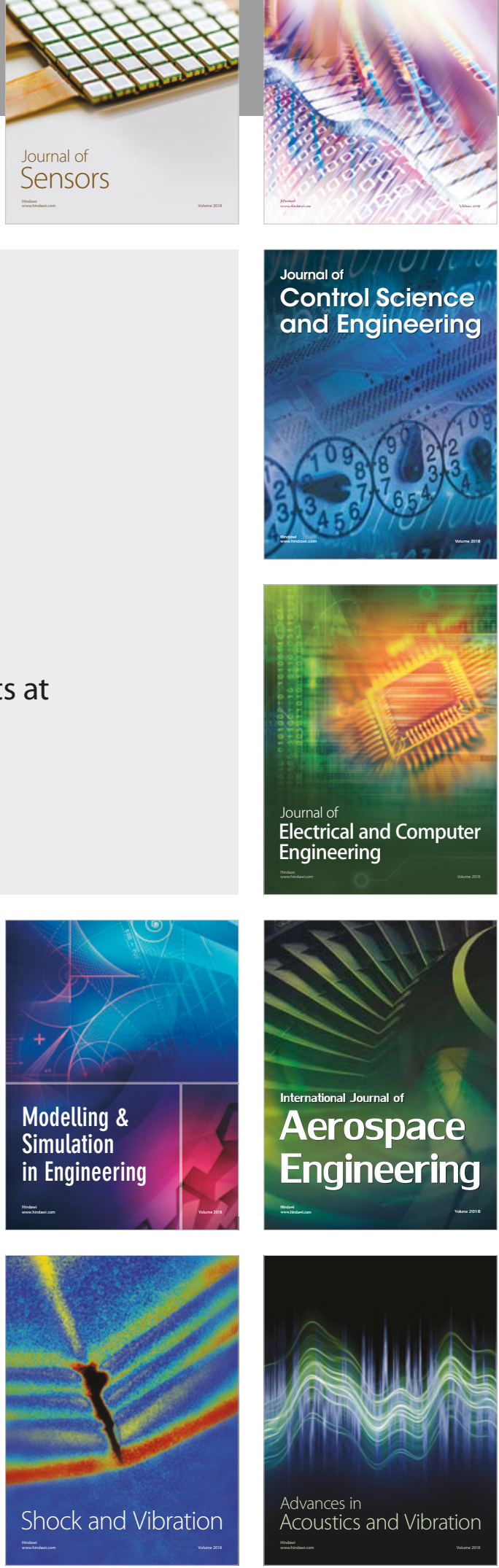